\title{
A preliminary study of semantics elicited by haptic stimulation of buttons by shape and haptic texture by using a single-key handheld device
}

\author{
Chao Yang Yang \\ Department of Industrial Design, \\ Tatung University \\ No. 40, Sec. 3, ZhongShan N. Rd., \\ Taipei City, 104, Taiwan R.O.C. \\ dillon.yang@gmail.com
}

\author{
Yu Ting Chen \\ Department of Industrial Design, \\ Tatung University \\ No. 40, Sec. 3, ZhongShan N. Rd., \\ Taipei City, 104, Taiwan R.O.C. \\ cyt811014@gmail.com
}

\author{
Chang Li Pao \\ Department of Industrial Design, \\ Tatung University \\ No. 40, Sec. 3, ZhongShan N. Rd., \\ Taipei City, 104, Taiwan R.O.C. \\ andy31201@gmail.com
}

\begin{abstract}
Effective designs compel customers to purchase products. Adding extra value to products by characterizing users' experiences can lead firms to greater market success. Haptic stimulation can be added to products to enhance product performance and consumer satisfaction. Handheld products not only integrate sensors, such as pressure sensors and fingerprint scanners, into their buttons but also feature materials, shapes, and physical feedback, all of which enrich users' haptic experience. In contrast to other sensory modalities, haptic sensors can detect various information types, such as pressure, weight, shape, and temperature, which are typically input in a single action. Products may induce various emotional responses depending on their shape and haptic feedback capabilities. Most studies have focused on the visual consistency of stimuli, but not semantic interference, between haptic modalities. This study investigated the relationship between the product semantic and affective response through cross-modal stimulation of button design. The results indicate that buttons with convex features result in high levels of arousal but low levels of valence, whereas buttons with an engraved square line result in the highest levels of valence. In the future, the event-related potential method will be used to test this study's applicability.
\end{abstract}

Keywords: button, feedback, haptic, emotional dimension

\section{INTRODUCTION}

Product shapes result from a designer's personal experience (Rajapakse, Jayasinghe, Tokuyama, Miyata, \& Marasinghe, 2009), and several studies have explored how design elements link to emotion. Most studies on emotional design have focused on the visual appearance of products. For instance, (Bar \& Neta, 2006) determined that consumers prefer rounded shapes over sharp shapes. The research has ranged from car exterior and interior appearance (Leder \& Carbon, 2005) to cellphone appearance (Ohkura, Konuma, Murai, \& Aoto, 2008). Streamlined designs are widely applied in furniture and consumer electronics industries to attract consumers' attention. However, vision is not the only sense involved in perceiving product quality. For instance, consumers first perceive a mobile device's shape and texture through touch when removing it from their pocket or purse. Haptic information guides the user when operating the product and conveys quality through the product's texture, shape, and material.

Tactile sensations have a profound psychological role; therefore, users store them in their long-term memory, and these directly affect consumer preferences (Krishna \& Morrin, 2007; Nagai \& Georgiev, 2011). Positive or negative emotional responses affect users' acceptance and use of a product (Jordan, 1998). Thus, the role of haptic sensation differs from that of visual sensory input in product design. However, the interaction between shape preferences and haptic sensations requires further investigation.

Most studies on shape preferences have compared sharp and rounded shapes. Differences in product appearance may affect users' emotional responses. Modern products require high haptic quality. This study explored the relationship between the functional button of a mobile device and users' emotional responses. 

handheld device.

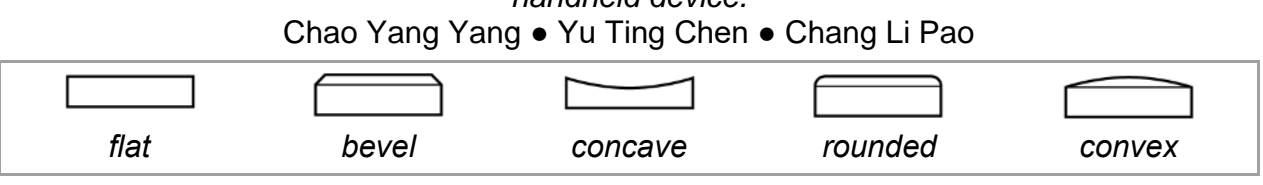

Figure 1: Button profile shapes

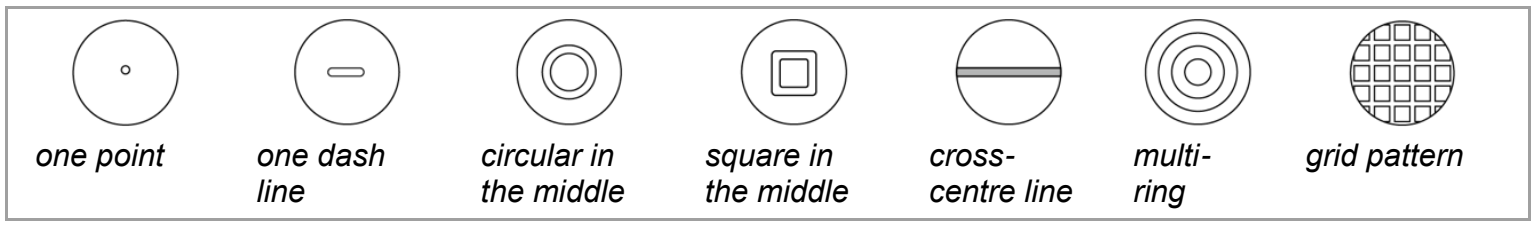

Figure 2: Top view of button patterns

\subsection{Button designs}

Single-button handheld products vary in finger and product contact surfaces. Button shape is not within the scope of this study. Button profile shapes are usually categorized into five types: flat, bevel, concave, round, and convex (Figure 1).

In addition to material differences, buttons adopt several types of texture. Top view of button patterns can be classified as indented, raised, nondense, and dense (Figure 2).

\subsection{Emotions}

User emotions are pivotal to product design (Alcántara, Artacho, González, \& García, 2005). Products arouse emotional responses in users and result in behavioral effects (Desmet \& Hekkert, 2007). Products not only act as functional objects but also play a role in making users feel happy, sad, reassured, or anxious (Marzano, 1998). To attract users, the product in the process of being experienced must cause the users to experience pleasure. In addition to its basic functionality and safety considerations, a product's ability to invoke positive emotions can enhance the user's feelings for it (Helander, 2002).

On the emotional dimension scale, common composition dimensions are "valence" and "arousal." Valence refers to the type of emotion induced by objects, whereas arousal indicates the degree to which such emotions are evoked. Russell (2003) introduced the concept of core affect combined with the psychological and neurological response formed by the circumplex model of affect. Emotional description is two dimensional; any emotion can be evaluated and explained using valence and arousal. The horizontal axis runs from pleasure to displeasure, whereas the vertical axis runs from activation to deactivation, and the interactions occur among various emotions.

\subsection{Haptic perception}

The eye receives stimulation, and the brain produces images and feelings. However, haptic sensations are intuitive reactions to a product.
Consumer purchase intention is affected by touch. For example, if a store does not allow consumers to touch its products, consumer purchase intention decreases.

Touch is a complex process. The aspects of touch that affect customer purchase intention include (1) horizontal scanning material, (2) product pressure sensitivity and hardness, (3) temperature, (4) weight, (5) overall appearance and volume, and (6) extension profile detection shape features (Lederman \& Klatzky, 1987).

\subsection{Aims}

In this study, we selected the style, vibration feedback, and button texture of a one-button cellphone as the key design parameters and investigated the emotions elicited by various button designs. In particular, we aimed to answer the following questions:

(i) What are the main features of the button design?

(ii) To what extent is emotional satisfaction elicited by the design features?

(iii) To what extent is emotional satisfaction elicited by the combinations of design features?

\section{METHODS}

The experimental process of this study included two phases. In the first phase, the design features of single-button handheld devices were analyzed. Relevant products on the market were collected and classified. In the second phase, the emotional cognition for buttons with various shapes and patterns was analyzed. The button design features were assessed using a self-assessed manikin (SAM) method. All procedures were designed to meet ethical standards for human participant research passed Research Ethics Office of National Taiwan University (Demirbilek \& Sener, 2003)(IRB no. 2016ES026). Respondents understood and agreed to the risks involved in the experiment before it was performed. 


\subsection{First phase: button features investigation}

In the first stage, the main features of a modern one-button device design were investigated; onebutton handheld devices were collected and the shape characteristics of their buttons were analyzed.

\subsubsection{Button design survey}

Various button designs were collected from shopping malls, consumer electronics retailers, and online stores. Products were collected between
November 2016 and January 2017. In total, 951 products were collected, of which 668 were consumer electronics products and 283 were home appliances. Product photographs and online screenshots were used for analysis; the frequencies at which the shape features were used were counted to rank features according to the use. The most widespread features were classified and drawn using Adobe Illustrator.

Table 1: Market survey results of button design in profile

\begin{tabular}{|c|c|c|c|c|c|c|}
\hline $\begin{array}{c}\text { Button side } \\
\text { features }\end{array}$ & flat & bevel & $\begin{array}{l}\square \\
\text { concave }\end{array}$ & round & convex & others \\
\hline $\begin{array}{l}\% \\
\text { (frequencies) }\end{array}$ & $\begin{array}{l}18.8 \% \\
(179)\end{array}$ & $\begin{array}{l}11.7 \% \\
(111)\end{array}$ & $\begin{array}{l}16.4 \% \\
(156)\end{array}$ & $\begin{array}{l}24.6 \% \\
(234)\end{array}$ & $\begin{array}{l}21.6 \% \\
(205)\end{array}$ & $\begin{array}{l}7.9 \% \\
(75)\end{array}$ \\
\hline
\end{tabular}

Table 2: Market survey results of button texture

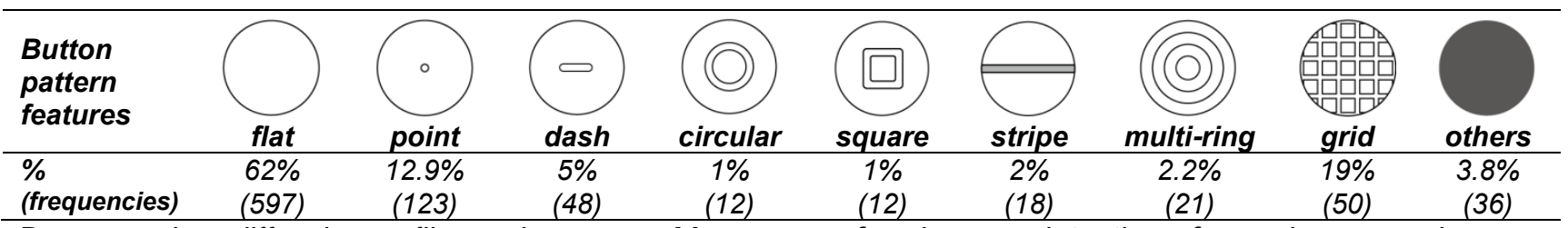

Button styles differ in profile and texture. Most buttons are flat, bevel, concave, rounded, or convex (Table 1) when viewed from the side and have circular, square, dash, dot, ring, and grid patterns when viewed from above. Textures include indented, raised features. Here, most buttons were circular $(57.6 \%)$. Rounded buttons were the most common $(24.6 \%)$, whereas bevelled buttons were the least common (11.7\%). Flat texture was the most used one (62\%) (Table 2), followed by gridpattern (19\%) and one-point (12.9\%) texture.

\subsection{Second phase: Emotional test of button designs}

In this phase, the cognitive emotional reactions to a set of redesigned parametric buttons (section 2.2.3.) were tested for valence and arousal. The three design features, side profile and two texture features (e.g. indentation and pattern), were selected as the independent variables. Shape was classified into five categories, namely flat, bevelled, concave, rounded, and convex, and texture into mixture of indentation and pattern. In total, 95 (19 textures $x 5$ side profiles) buttons were designated as stimuli.

\subsubsection{Emotion measurements}

Emotions elicited through physiological stimulation can be mapped on the circumplex model of affect (Russell, 2003) according to valence and arousal. The $X$ and $Y$ dimension indicated affect valence (pleasure) and arousal (excitement) of the stimuli, respectively. We used the collocation SAM method

for image detection for valence and arousal measurement (Bradley \& Lang, 1994).

\subsubsection{Participants}

70 (35 males and 35 female) right-handed Taiwanese graduates with normal vision and tactile sensitivity were selected to participate. Those with visual dysfunction or a history of neurological or cognitive dysfunction were excluded. Tactile sensitivity slows with age (Kleinman \& Brodzinsky, 1978); thus, our participants were aged only 20-30 years. The participants were required to confirm whether their mental state was normal and provide their medication, alcohol, caffeine, exercise, and sleep statuses before the experiment to eliminate result bias.

\subsubsection{Stimulus design}

In the first phase, 19 button designs were used. All were circular but varied in profile (flat, bevelled, concave, rounded, or convex) and texture (indented or raised), according to the market survey.

\subsection{Stimulus reproduction}

Stimuli were designed using RHINO 3D and threedimensionally prototyped using an ATOM 3D printer for initial checking. Confirmed designs were reproduced in finer quality by using a Roland SRM20 computer numerical controlled milling machine.

\subsection{Button mechanical setting}


Chao Yang Yang • Yu Ting Chen • Chang Li Pao

The stimuli were fixed onto membrane switches. The click threshold of the membrane switches was set at 60-300-g peak force to provide comfortable feedback. After pressing the button, the membrane

\subsection{Emotion evaluation environment setting and procedure}

The objective during this stage was to confirm whether the participants consistently pressed the stimuli. The experimental environment (Figure 3) was designed in which six reproduced stimuli, including one neutral stimulus (T1) and five other stimuli, were fitted to a round plate placed on a 70cm-high desk, approximately $20-30 \mathrm{~cm}$ from the participants. Nineteen stimulus plates were prepared and tested in turn. Four plates were fabricated, and the evaluator rotated the plate so that each blindfolded participant could touch each stimulus in turn. The evaluator subsequently asked the participants to evaluate the valence and arousal of the stimuli. Stimulus plates were changed and tested randomly.
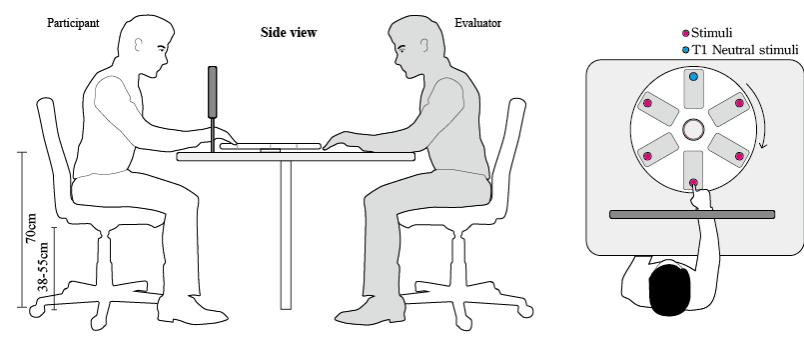

Figure 3: Experimental environment

\section{RESULTS}

We investigated the emotional responses to button profile and texture by using an emotional cognitive questionnaire. As results, button texture significantly affect valence and arousal $(p<0.05)$ whereas profile and combined profile and texture does not affect valence or arousal ( $p>0.05)$ interactively. Therefre, a diagram is drawn to show the button texture distribution in which valence is set as the horizontal axis and arousal as the vertical axis. In addition, 3 is the neutral score, scores above which are positive but below which are negative. In a paired sample $t$ test, T3, T4, T5, T6, T7, T8, T9, T10, T12, T13, T14, T15, and T18 were significantly similar to $\mathrm{T} 1$ (i.e., the plain flat design; $p \leq 0.05)$ for valence, whereas T2, T3, T4, T5, T6, T7, T8, T9, T10, T11, T12, T13, T14, T15, $\mathrm{T} 16, \mathrm{~T} 18$, and $\mathrm{T} 19$ were significantly similar to $\mathrm{T} 1$ $(p \leq 0.05)$ for arousal.

Most button designs stimulated emotion to a greater degree than the plain button design. T1 sprang back to its initial position. Because stroke length affects the clicking experience, it was set within the ranges of $0.1-0.5 \mathrm{~mm}$ (flat type) and 0.6$1.5 \mathrm{~mm}$ (tactile type).

resulted in low levels of emotional activation and close-to-neutral pleasure. T4, T15, T16, and T19 resulted in high levels of arousal and valence, whereas T5, T7, T9, and T13 resulted in high levels of arousal and low levels of valence. T4 and T5 evoked opposing emotional states for a design that was identical except that one has a concave texture and the other has a convex texture. Lowlevel valence buttons, including T11 (square) and T13 (circular), possessed convex features, which stimulated similar arousal levels but different valence levels. Although T11 (circular and convex) and T10 (circular and concave) evoked similar valence levels, T11 evoked higher arousal levels.

\section{CONCLUSION}

Button design is a key consideration in the mobile device industry. This paper provides evidence supporting the significance of kansei engineering in product design because participants exhibited different perceptions toward different button designs. Significant differences were observed for button profiles and texture (indent vs. raised; $p=$ $0.00)$. The results provide a reference to designers for integrating emotional concepts in which texture may contain emotional messages into product design.

This study is the first in a series on the affective qualities of buttons; a study on haptic event-related potential validation will be presented in the future. In the next stage, buttons with high levels of arousal with high- and low-level valence stimuli will be selected. Because of the strength of emotion (arousal > 3) that they evoke, T5, T7, T9, and T13 will be selected to invoke negative emotions, whereas T4, T15, T16, and T18 will be selected to invoke positive emotions. In addition to T1, T11 will be used as a neutral stimulus because of the high level of arousal and negligible valence bias that it invokes.

\section{ACKNOWLEDGEMENT}

The authors gratefully acknowledge the financial support of the Ministry of Science and Technology project grant: 106-2410-H-036 -005. 


\section{REFERENCE}

Alcántara, E., Artacho, M., González, J., \& García, A. C. (2005). Application of product semantics to footwear design. Part l-Identification of footwear semantic space applying diferential semantics. International Journal of industrial ergonomics, 35(8), 713-725.

Armstrong, G., Kotler, P., Harker, M., \& Brennan, R. (2011). Marketing: an introduction (Vol. 10): Pearson NJ.

Bar, M., \& Neta, M. (2006). Humans prefer curved visual objects. Psychological science, 17(8), 645-648.

Bradley, M. M., \& Lang, P. J. (1994). Measuring emotion: the self-assessment manikin and the semantic differential. Journal of behavior therapy and experimental psychiatry, 25(1), 49-59.

Demirbilek, O., \& Sener, B. (2003). Product design, semantics and emotional response. Ergonomics, 46(13-14), 1346-1360.

Desmet, P., \& Hekkert, P. (2007). Framework of product experience. International Journal of Design, 1(1).

Glen, U., \& Hauser, J. (1993). Design and marketing of new products: Prentice Hall, Englewood Cliffs, NJ.

Helander, M. G. (2002). Hedonomics-affective human factors design. Paper presented at the Proceedings of the Human Factors and Ergonomics Society Annual Meeting.

Jordan, P. W. (1998). Human factors for pleasure in product use. Applied Ergonomics, 29(1), 2533.

Khalid, H. M. (2006). Embracing diversity in user needs for affective design. Applied ergonomics, 37(4), 409-418.

Kleinman, J. M., \& Brodzinsky, D. M. (1978). Haptic Exploration in Young, Middle-aged, and Elderly Adults 1 2. Journal of Gerontology, 33(4), 521527.

Krishna, A., \& Morrin, M. (2007). Does touch affect taste? The perceptual transfer of product container haptic cues. Journal of Consumer Research, 34(6), 807-818.

Leder, H., \& Carbon, C. C. (2005). Dimensions in appreciation of car interior design. Applied Cognitive Psychology, 19(5), 603-618.

Leder, H., Tinio, P. P., \& Bar, M. (2011). Emotional valence modulates the preference for curved objects. Perception, 40(6), 649-655.

Lederman, S. J., \& Klatzky, R. L. (1987). Hand movements: A window into haptic object recognition. Cognitive psychology, 19(3), 342368.

Marzano, S. (1998). Creating value by design (Vol. 1): V \& K Publishing.

Nagai, Y., \& Georgiev, G. V. (2011). The role of impressions on users' tactile interaction with product materials: An analysis of associative concept networks. Materials \& Design, 32(1), 291-302.

Norman, D. A. (2004). Emotional design: Why we love (or hate) everyday things: Basic Civitas Books.

Ohkura, M., Konuma, A., Murai, S., \& Aoto, T. (2008). Systematic study for "kawaii" products (the second report)-commpmrison of "kawaii" colors and shapes. Paper presented at the SICE Annual Conference, 2008.

Palumbo, L., Ruta, N., \& Bertamini, M. (2015). Comparing angular and curved shapes in terms of implicit associations and approach/avoidance responses. PloS one, 10(10), e0140043.

Rajapakse, R. J., Jayasinghe, H., Tokuyama, Y., Miyata, K., \& Marasinghe, A. (2009). The effect of variable base on perceiving gender kansei of $3 D$-shape. Paper presented at the Biometrics and Kansei Engineering, 2009. ICBAKE 2009. International Conference on.

Russell, J. A. (2003). Core affect and the psychological construction of emotion. Psychological review, 110(1), 145-172. 